\title{
O ARQUIVOS BRASILEIROS DE CIRURGIA DIGESTIVA - ABCD COMPLETA 35 ANOS E INICIA UM NOVO CICLO
}

\author{
THE BRAZILIAN ARCHIVES OF DIGESTIVE SURGERY COMPLETES 35 YEARS AND A NEW CYCLE BEGINS
}

Nelson Adami ANDREOLLO ${ }^{\oplus}{ }^{\circ}$, Francisco TUSTUMI ${ }^{\circ}$, José Eduardo de AGUILAR- NASCIMENTO ${ }^{\circ}$

$\mathrm{O}$ $A B C D-A R Q U I V O S$ Brasileiros de Cirurgia Digestiva - em 2021 - completa 35 anos de publicações ininterruptas. Foi idealizado pelo Professor Henrique Valter Pinotti, seu fundador e primeiro editor, em $1986^{1}$, e teve seu primeiro número publicado ainda no início daquele ano (Figura 1). Ao longo de seus anos, o ABCD - como bem descrito nas palavras do Professor Osvaldo Malafaia ${ }^{4}$, seu editor durante 20 anos (2001-2021) - seguiu uma "TRAJETÓRIA VITORIOSA!".

O ABCD publicou trabalhos científicos produzidos por autores e pesquisadores brasileiros e do exterior, contribuindo para disseminar os avanços da cirurgia digestiva, gastroenterologia, endoscopia digestiva e nutrição. Durante os 35 anos, foram publicados aproximadamente 1650 trabalhos científicos ${ }^{5}$.

Nosúltimos anos o $A B C D$ recebeu submissões de trabalhos nacionais e de trabalhos científicos de autores de vários outros países, como Estados Unidos, Inglaterra, Espanha, França, Bélgica, Alemanha, México, Chile, Argentina, Peru, Cuba, Índia, Arábia Saudita, Turquia, China, Iran e outros, evidenciando o seu ganho em prestígio e reputação internacional ${ }^{5}$.

Foram anos de enormes esforços e contínua dedicação de seus editores, editores associados e membros do Conselho Editorial, analisando trabalhos científicos, orientando seus autores para que fossem publicados e divulgados o melhor da cirurgia digestiva do país e do mundo.

O Professor Henrique Valter Pinotti, com a sua percepção futurística de educador, pesquisador e excelente cirurgião, foi também o idealizador do Colégio Brasileiro de Cirurgia Digestiva (CBCD), fundado em 1988. OCBCD, desde então, com a missão de congregar cirurgiões do aparelho digestivo, por meio dos seus Presidentes e das Diretorias, tem se dedicado ao aprimoramento científico de seus membros, na defesa do exercício profissional e condições de trabalho, no sentido de oferecer segurança e qualidade ao profissional no desempenho de sua função ${ }^{8}$.

No início da década de 1990, o Professor Henrique Valter Pinotti, integrou a Revista $A B C D$ ao $C B C D$, objetivando,

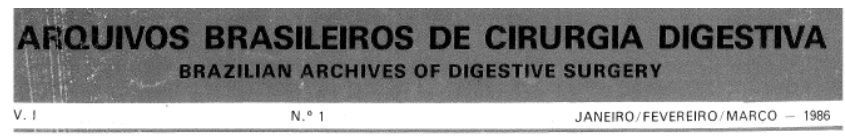

CONTENTS ISSN 0102-6720

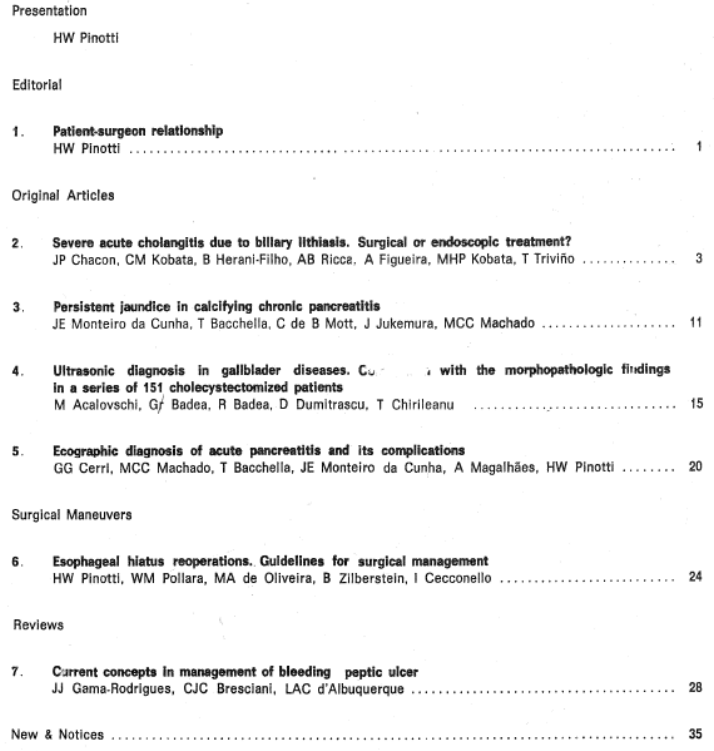

Figura 1 - Artigos que formavam o número 1, volume 1, Janeiro/ Fevereiro/ Março, 1986.

também atingir cirurgiões jovens e residentes de Cirurgia do Aparelho Digestivo, contribuindo assim na sua formação, para bem tratar os doentes mais graves e complexos, bem como executar com sucesso intervenções de grande porte cirúrgico. Além disso, divulgar as pesquisas oriundas dos Cursos de Pós-Graduação ${ }^{5,6,7}$.

Professor Titular de Cirurgia, Departamento de Cirurgia, Faculdade de Ciências Médicas da Universidade Estadual de Campinas - Unicamp, Campinas - São Paulo, Brasil

2 Departamento de Gastroenterologia, Hospital de Clínicas da Faculdade de Medicina da Universidade de São Paulo - HCFMUSP, São Paulo - São Paulo, Brasil

${ }^{3}$ Professor Titular, Departamento de Clínica Cirúrgica, Faculdade de Medicina da Universidade Federal de Mato Grosso (UFMT) - Cuiabá, Mato Grosso, Brasil. Diretor do Curso de Medicina do Centro Universitário de Várzea Grande (UNIVAG)

Como citar esse artigo: Andreollo NA, Tustumi F, Aguilar- Nascimento JE. O Arquivos Brasileiros de Cirurgia Digestiva - ABCD completa 35 anos e inicia um novo ciclo. ABCD Arq Bras Cir Dig. 2021;34(4):e1623. https://doi.org/10.1590/0102-672020210002e1623

Correspondência:

Nelson Adami Andreollo

Email: nandreollo@hotmail.com
Fonte de financiamento: nenhum.

Conflito de interesse: não.

Recebido: 02/11/2021

Aceito: 25/11/2021 
O seus editores, em ordem cronológica, foram:

- 1986 a 1990-Conselho Editorial - Professores Angelita Habr-Gama, Bruno Zilberstein, Ivan Cecconello, Joaquim Gama-Rodrigues e Marcel Cerqueira Cesar Machado.

- 1991 a 2000 - Professor Bruno Zilberstein

- 2001 a 2021 - Professor Osvaldo Malafaia

Em 2021, um novo Corpo Editorial tem por desafio manter a qualidade e ampliar o prestígio da Revista. O novo Corpo Editorial está sendo reformulado e irão integrar cirurgiões especialistas nacionais e do exterior, de áreas específicas, tais como Cirurgia Digestiva (doenças benignas e oncológicas), Cirurgia da Obesidade e Metabólica, Cirurgia do Fígado, Vias Biliares e Pâncreas, Videocirurgia e Robótica, Coloproctologia, Transplante de Órgãos, Nutrição e Cuidados Perioperatórios

O Professor Osvaldo Malafaia, sem dúvida, foi o editor quem contribuiu para a Revista atingisse o patamar atual de visibilidade, indexando-a ao Scielo - Scientific Electronic Library Online (desde 2007), ao Medline/PubMed (desde 2012), ao PubMed Central (desde 2014) e as demais bases de indexação mundial (Scopus/Scimago, Web of Science - Emerging Sources Citation Index (ESCI), Google Scholar, LILACS e DOAJ) 3,4,5,6.

Dessa forma, a atual Diretoria do CBCD reconheceu e designou o Professor Osvaldo Malafaia como EDITOR EMÉRITO da Revista Arquivos Brasileiros de Cirurgia Digestiva - ABCD.

Todos os Presidentes e suas respectivas Diretorias do Colégio Brasileiro de Cirurgia Digestiva, direta e indiretamente contribuíram, e acreditaram nos investimentos necessários, para o sucesso da Revista. A seguir, os presidentes do CBCD (1988 - 2021):

- Professor Henrique Walter Pinotti, fundador e primeiro presidente - 1988/1990 - (Universidade de São Paulo - USP - São Paulo - SP);

- ProfessorLuizSérgio Leonardi-1991/1992-(Universidade Estadual de Campinas - UNICAMP - Campinas - SP)

- Professor Edmundo Machado Ferraz - 1993/1994 - (Universidade Federal de Pernambuco - UFPE Recife - PE)

- Professor Luiz Rohde - 1995/1996 - (Universidade Federal do Rio Grande do Sul - UFRS - Porto Alegre - RS);

- Professor Alcino LazaroSilva-1997/1998-(Universidade Federal de Minas Gerais - UFMG - Belo Horizonte - MG);

- Professor Osvaldo Malafaia-1999/2000-(Universidade Federal do Paraná - UFPR - Curitiba - PR);

- Professor Joaquim José Gama-Rodrigues - 2001/2002 - (Universidade de São Paulo - USP - São Paulo - SP);

- Professor Paulo Roberto Rocha Savassi - 2003/2004 (Universidade Federal de Minas Gerais - UFMG - Belo Horizonte - MG)

- Professor Julio Cesar Uilli Coelho - 2005/2006 (Universidade Federal do Paraná - UFPR - Curitiba - PR)

- ProfessorAngelita Habr-Gama-2007/2008-(Universidade de São Paulo - USP - São Paulo - SP)

- Professor Nelson Adami Andreollo - 2009/2010 (Universidade Estadual de Campinas - UNICAMP Campinas - SP)

- Professor Cleber Dario Pinto Kruel - 2011/2012 (Universidade Federal do Rio Grande do Sul - UFRS - Porto Alegre - RS)

- Professor Ivan Cecconello-2013/2014 - (Universidade de São Paulo - USP - São Paulo - SP)

- Professor BrunoZilberstein-2015/2016-(Universidade de São Paulo - USP - São Paulo - SP)

- Professor Nicolau Gregori Czeczko - 2017/2018 (Universidade Federal do Paraná - UFPR - Curitiba - PR)

- Professor Delta Madureira Filho-2019/2020-(Universidade Federal do Rio de Janeiro - Rio de Janeiro - RJ)
- Professor Luiz Augusto Carneiro D’Albuquerque 2021/2022 - (Universidade de São Paulo - USP - São Paulo - SP)

- Professor Antonio Carlos Ligocki Campos - 2023-2024 (eleito) - (Universidade Federal do Paraná - UFPR Curitiba - PR)

Os editores atuais do $A B C D$ irão manter as orientações dos editores anteriores, que é de divulgar a cirurgia digestiva, além manter e ampliar o universo de conquistas reunidas pelo Professor Osvaldo Malafaia.

$O A B C D$ irá continuar divulgando os progressos na etiopatogenia e diagnóstico das doenças, as pesquisas experimentais relacionadas ao aparelho digestivo, os cuidados perioperatórios, cirurgia tradicional, as novas técnicas cirúrgicas, a cirurgia experimental, as revisões sistemáticas e metaanálises, a videocirurgia, a cirurgia bariátrica e metabólica, a cirurgia oncológica no aparelho digestivo, nutrição em cirurgia, a cirurgia endoscópica e mais recentemente a cirurgia robótica.

Durante os últimos 35 anos, a Revista circulou na forma impressa entre os cirurgiões e membros do Colégio Brasileiro de Cirurgia Digestiva. A partir de 2007, passou a ser divulgada totalmente bilingue e também online, de acesso livre para obtenção de todos os artigos publicados e sem custo, via Scielo, Medline/PubMed e PubMed Central, em todas as edições. Com o acesso atual facilitado às bases indexadoras no mundo todo e o amplo acesso a tablets, celulares, notebooks e microcomputadores, a partir de 2022, a Revista passará a ser divulgado somente online, nas bases indexadoras. Ademais, com o intuito de expandir a internacionalização da revista, todas as publicações serão no idioma inglês.

Considerando o crescente interesse do público nãoespecialista nas temáticas científicas e a necessidade de contribuição social, o atual corpo editorial da revista $A B C D$ se propõe a se aproximar do público geral, sem perder, no entanto, a atenção ao seu público especialista. Dia após dia crescem as tecnologias de comunicação em rede, as quais podem ser uma eficiente ferramenta para promover a circulação de informações.

Assim, a partir de 2022 o ABCD se propõe a se inserir nas chamadas redes sociais: Facebook, Twitter, YouTube, Instagram, Wikipedia e blogs. As redes sociais são plataformas populares, que abrem a possibilidade de ampliar a distribuição de notícias, não apenas de interesse do médico especialista, mas de interesse do público leigo ${ }^{2}$. Dessa forma, o $A B C D$ objetiva contribuir para o ensino e disseminação das pesquisas e da medicina baseada em evidências.

Novos revisores nacionais e internacionais participarão do processo de peer-review de cada artigo submetido à revista. O corpo de revisores cobrirá todas as áreas do conhecimento em aparelho digestivo, nas suas diversas sub-especialidades, para que cada manuscrito seja bem avaliado e que as revisões contribuam de forma notória para o aprimoramento do artigo, antes de sua publicação. Nesse cenário, a participação dos revisores internacionais será crucial para o amplo intercâmbio de conhecimentos científicos.

Buscando facilitar o processo de submissão e a expansão do $A B C D$, o endereço eletrônico da revista será reformulado para 2022. O novo site trará recursos para favorecer a submissão de novos manuscritos pelos autores e para que o acesso ao conteúdo da revista seja amplamente disseminado.

Desse modo, a pretensão dos atuais editores é de consolidar as conquistas obtidas ao longo dos 35 anos de $A B C D$ e de ampliar a disseminação da ciência internacionalmente e nos mais diversos públicos, especialistas e não-especialistas. Assim, o $A B C D$ irá estender sua importância social e contribuir para 
o avanço e propagação da pesquisa na cirurgia do aparelho digestivo, no Brasil e no mundo, e consequentemente, ganhará mais impacto, prestígio e reputação.

\section{REFERÊNCIAS}

1. Andreollo NA, Cecconello I, Kruel CDP, Malafaia O. 25 years of $A B C D$ - surgeon-patient relationship in the past and present. ABCD Arq Bras Cir Dig. 2011; 24(4): 259-261. doi.org/10.1590/ S0102-67202011000400002.

2. Araujo RF. Scientific digital marketing and altmetrics for academic journals: from visibility to engagement. Perspect Ciênc Inf. 2015;20(3):67-84. doi.org/10.1590/1981-5344/2402
3. Andreollo NA, Malafaia O. Silver jubilee of the $A B C D$. $A B C D$ Arq Bras Cir Dig. 2011;24(4):257-58. doi.org/10.1590/S010267202011000400001.

4. Kruel C, Malafaia O.ABCD included in Medline/Pubmed. ABCD Arq Bras Cir Dig.2012(1):1-1. doi:10.1590/s0102-67202012000100001.

5. Malafaia O. The ABCD from 1986 to 2021: Victorious trajectory! ABCD Arq Bras Cir Dig. 2021;34(2):e1609. doi: https://doi.org/ 10.1590/0102-672020210002e1609

6. Malafaia O, Zilberstein B, Pinheiro MCP, Cunha LC, Savassi-Rocha $P C$. The Journal ABCD going to the SciELO. ABCD Arq Bras Cir Dig. 2003;16(3):103-104.

7. Zilberstein B, Gama-Rodrigues JJ, Malafaia O. New Trends. ABCD Arq Bras Cir Dig. 2001;14(1):1-1.

8. Zilberstein B. CBCD: 25 years later! Arq Bras Cir Dig. 2015;28(1):1. doi: 10.1590/S0102-67202015000100001. 